\title{
Automated Search for Gravitational Lensing Arcs and Interacting Galaxies in the Red Sequence Survey
}

\author{
P. S. Chiang, S. Y. Chen, W. Ip, and W. P. Chen \\ Gratuate Institute of Astronomy, National Central University, Jhong-Li 32054, Taiwan \\ email: pschiang@astro.ncu.edu.tw
}

\begin{abstract}
We have developed a robust and efficient algorithm to identify gravitational lensing arcs and interacting galaxies. The algorithm has been applied to the Red Sequence Survey II (RCS II) data, and we demonstrate a few examples here.
\end{abstract}

Our algorithm has two main parts: (1) Moment analysis to calculate the best-fit ellipse of an object. The second moments are $I_{\min }=\pi a b^{3}$ and $I_{\max }=\pi a^{3} b$, where $I$ is the moment and $a, b$ are major and minor axes which define the best-fit ellipse. Those objects with morphology similar to their best-fit ellipses are excluded. (2) Recognition and parameterization of an arc by Thomas-Chen (Thomas and Chen, 1989, Computer Vision, Graphics and Image Processing, 45, 362) and Landau (Landau, 1987, Computer Vision, Graphics, and Image Processing, 38, 317) arc-fitting algorithms. Our algorithm is useful on identification of arcs, interacting, and irregular galaxies.

We analyzed 16 RCS II fields and identified 127 candidates, out of which 50 show distorted or clumpy structures and 77 are apparent galaxy pairs. Three such cases are demonstrated in Fig. 1. Most false detections are caused by bad images, bright stars, galaxies, and even spiral arms of galaxies. Our algorithm finds arc-like structures without any bias on over-density regions. For example, the gravitational lensing arc candidate in Fig. 1 is not in an over-density region, so may be difficult to find by visual inspection. Another niche of our algorithm is its efficiency to deal with data with large sky coverage.
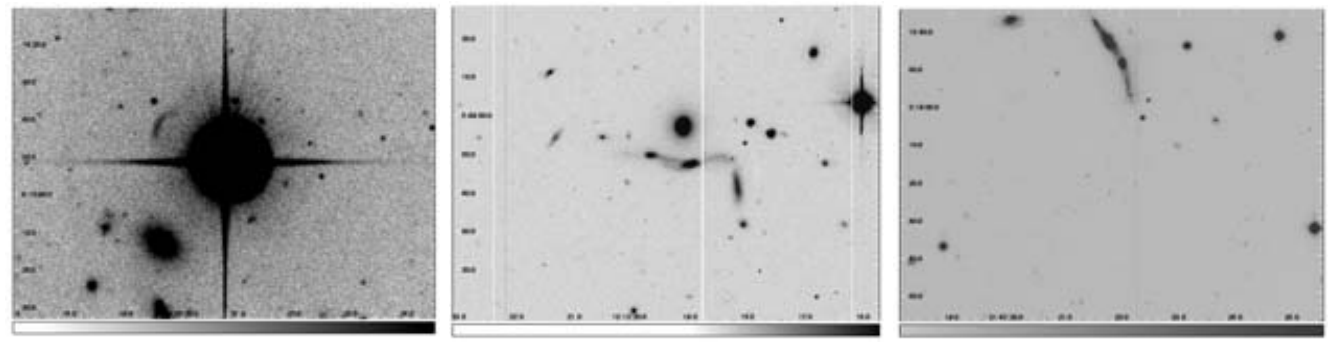

Figure 1. (Left): The candidate of gravitational lensing arc $(\alpha: 16 \mathrm{~h} 54 \mathrm{~m} 19.6 \mathrm{~s}, \delta: 06 \mathrm{~d} 14 \mathrm{~m} 42 \mathrm{~s})$. The arc is located to the upper left of the bright star. (Middle): Interacting galaxies with tidal tails $(\alpha: 15 \mathrm{~h} 12 \mathrm{~m} 19.0 \mathrm{~s}, \delta: 05 \mathrm{~d} 48 \mathrm{~m} 46 \mathrm{~s})$. (Right): A chain-shaped galaxy $(\alpha: 21 \mathrm{~h} 47 \mathrm{~m} 21.8 \mathrm{~s}, \delta:-02 \mathrm{~d} 18 \mathrm{~m} 46 \mathrm{~s})$, similar to those discovered in the Hubble Deep Field (for example, Elmegreen et. al, 2005, ApJ, $631,85)$. 